\title{
Distributed Generation Planning Strategy with Load Models in Radial Distribution System
}

\author{
Deependra Singh, Devender Singh and K S Verma
}

\begin{abstract}
This paper proposes a new approach for distributed generation (DG) planning from the perspective of a distribution company (disco). Optimal sizing and siting decisions for distributed generator is obtained through different approaches: Power loss minimization, System MVA minimization, System cost minimization and System Energy loss minimization. The methodology adopted permits the planner to decide optimal location and size of DG with compromise between Power loss, System MVA, System cost and System energy loss. Normally a constant power (real and reactive) load model is assumed in most of the studies. It is shown that load models can significantly affect the optimal location and sizing of DG resources in distribution systems. A comparative study of optimal location and size of distributed generator provided by installing DG resources with different type of loads models has been performed.
\end{abstract}

Index Terms-Distributed generation (DG), load models, distribution system.

\section{INTRODUCTION}

Distribution company(disco) planners continuously exercising to develop new planning strategies for their network to fulfill load growth requirement and provide their customers with flexible and reliable electric power. The importance of harnessing energy savings and minimizing environmental impacts has been providing the interest in development of distributed generation (DG). Recent studies have predicted that by year 2010, distributed generation will account for up to $25 \%$ of all new generation. Normally, DGs are integrated in the existing distribution system and the planning studies have to be performed for optimal location and size of DGs to yield maximum benefits. Electric utilities (or energy serving entities) try to provide reliable supply of electric power to their customers. Maximum benefits require both low cost and sufficient supply availability.

Y. G. Hegazy et al. [1] evaluated the effect of DGs controlled by the customer on system power capacity to satisfy the total system load and predicted the average amount of unsupplied demand for a given year. The authors used Monte Carlo approach to model the operating histories of the installed distributed generators. Walid El-Khattam et al. [2, 3] proposed a method of solving distributed generation planning problem (location and size) in different

Deependra Singh is Assistant Professor in Electrical Engineering Department, Kamla Nehru Institute of Technology, Sultanpur, UP, INDIA.

Devender Singh is Reader in Electrical Engineering Department, Institute of Technology, Banaras Hindu University, Varanasi, UP, INDIA.

K S Verma is Professor in Electrical Engineering Department, Kamla Nehru Institute of Technology, Sultanpur, UP, INDIA utility scenarios as an optimization problem. The objective function was based on supply-demand chain which aimed to minimize the investment and operating costs of local candidate DGs, payments towards purchasing the required extra power by the DISCO, payments toward loss compensation services, as well as the investment cost of other chosen new facilities for different market scenarios.

Caisheng Wang et al. [4] proposed an analytical method to determine the best location of candidate DGs for minimum loss configuration. A rigorous analysis for uniformly distributed, centrally distributed, and increasingly distributed loads has been carried out for constant and time varying loads on a feeder. The proposed approach was non-iterative unlike power flow programs. Therefore, there is no convergence problem involved, and results could be obtained quickly. However, authors have indicated that other constraints such as voltage and line limits may affect the DG placement.

Víctor H. Méndez Quezada et al. [5] discussed such knowledge gap, through the analysis of the impact of DG on distribution losses by studying different scenarios with several DG penetration and concentration levels. Losses are evaluated on an annual basis. Different DG technologies, such as CHP, wind power, and photovoltaic cells, are modeled. For each type of technology, different penetration levels, corresponding with different amounts of DG installed capacity, and different concentration levels, corresponding with several DG units connected along the feeder, are studied.

Paul M. Sotkiewicz et al. [6] proposed a method for the allocation of fixed (capital and non-variable operation and maintenance) costs at the medium voltage (MV) distribution level. This method is derived from the philosophy behind the widely used MW-mile methodology for transmission networks that bases fixed cost allocations on the "extent of use" that is derived from load flows. The "extent of use" is calculated by multiplying the total consumption or generation at a bus bar by the marginal current variations, or power to current distribution factors (PIDFs) that an increment of active and reactive power consumed, or generated in the case of distributed generation, at each bus bar, produces in each circuit.

Load models are well known in stability studies [7]-[10]. These studies were normally aimed towards voltage or frequency dependent representation of load especially for dynamic or static stability studies of power systems. Exhaustive review of load model to be used for power flow and dynamic studies have been presented in [8]. The effect of load models in planning studies was demonstrated in optimal capacitor placement / switching by D.T. Rizy et al. 
[11] in a distribution system and by Stefan Arnborg et al. [12] in under-voltage load shedding studies. The authors demonstrated that consistent with the modeling of feeder load as constant power, it is expected that the switching of capacitor bank to improve the power factor results in decreased real and reactive power injection at the substation. This decrease in real and reactive power injections is due to improved voltage profile. In fact the measured real and reactive power injections increase. In this case, contrary to the constant power load modeling, a reduction in real and reactive power injection reflecting the reduced line losses was not observed at the substation. An assumption of constant power load modeling leads to general (mis)understanding of reduced power injection due to reduced losses. Analysis of the experimental results [12] using voltage sensitive load model shows that while the feeder losses are reduced following the capacitor placement; the attendant improvement in voltage profile results in an increase in loads that exceeds the amount of loss reduction.

In context of optimization, problem of DG placement is similar in nature to that of capacitor placement discussed above. Most of the planning methods invariably use power flow programs which normally utilize constant real and reactive power load model representation. It is observed from the literature review that load models were not included in planning the location and size, and calculating the said indices except T. Gozel et al. [13] who have used analytical approach considering the load model to show that the location does not change as significantly as size of DG. However, the analysis does not take the constraints of voltage and line limits and therefore has only instructive value.

Maximizing reliability can also be a criterion for seeking optimal DG location. Dan Zhu et al. [15] has done a reliability analysis based on set theory and discussed how the system load and additional generation (provided by DG) affect the system reliability. The manner in which these locations vary as a function of time-varying loading is considered. However analysis does not considered system energy loss minimization with time-varying loading.

Rodrigo Palma-Behnke et al. [16] presented a novel day-ahead energy acquisition model for a distribution company (disco) in a competitive market based on Pool and financial bilateral contracts. The market structure encompasses wholesale generation companies, distributed generation (DG) units of independent producers, DG units owned by the disco, and load curtailment options. Thus, while satisfying its technical constraints, the disco purchases active and reactive power according to the offers of DG units, customers, and the wholesale market.

Energy losses in distribution systems are generally estimated rather than measured, because of inadequate metering in these systems and also due to the high cost of data collection. These estimations are generally based on some rules of thumb. P S Nagendra Rao et al. [17] presented the results of a joint investigation undertaken in collaboration with a local utility to study this issue. Based on data collected from feeders specially instrumented for this purpose, true losses in some primary and secondary feeders are obtained. These losses are compared with the estimated losses obtained by the methods presently in use.
In this paper, a detailed analysis for DG planning is made on the basis of Power loss minimization, System MVA minimization, System cost minimization and System Energy loss minimization. Extent up to which load models can affect the location and size of the DGs in such studies has also been investigated. The voltage dependent load models for residential, industrial and commercial loads are adopted from [8]. A 38-bus radial distribution system from [11] is utilized for demonstrating the results. In the practical situation, loads are not explicitly residential, industrial, and commercial; rather load class mix may be seen by distribution system depending on the nature of area being supplied. Therefore, a load class mix of residential, industrial and commercial load is also investigated. It is also investigated load is a time-varying in nature. Therefore it is included in system energy loss analysis.

\section{LOAD MODELS AND LOAD PROFILE}

To quantify the effect of various load models on distributed generation planning a 16-bus distribution system is adopted (Fig. A1). The data for p.u. line impedances, load data and the line MVA limits are given in Appendix (Table A1). The effects of selected voltage dependent load models are investigated in different planning scenarios (test cases). Practical voltage dependent load models i.e. residential, industrial, and commercial given in [7] have been adopted for investigations. The load models can be mathematically expressed as

$$
P_{i}=P_{0 i} V_{i}^{\alpha}
$$

$$
Q_{i}=Q_{0 i} V_{i}^{\beta}
$$

Where, $P_{i}$ and $Q_{i}$ are real and reactive power at bus $i$, $P_{0 i}$ and $Q_{0 i}$ are active and reactive operating point at bus $i$, $V_{i}$ is voltage at bus $i$, and, $\alpha$ and $\beta$ are real and reactive power exponents. In a constant power model conventionally used in power flow studied $\alpha=\beta=0$ is assumed. The values of the real and reactive exponents used in the present work for industrial, residential and commercial loads are give in Table I [8].

\section{TABLE I}

Load Types and exponent values

\begin{tabular}{lll}
\hline Load Type & $\alpha$ & $\beta$ \\
\hline Constant & 0 & 0 \\
\hline $\begin{array}{l}\text { Industrial } \\
\text { Load }\end{array}$ & 0.18 & 6.00 \\
\hline Residential & 0.92 & 4.04 \\
\hline Commercial & 1.51 & 3.40 \\
\hline
\end{tabular}

During investigations the comparison of constant power load model assumption with the practical load models are emphasized. While investigating effect of residential load the 38-node system was assumed to be supplying residential consumers only (all loads are residential type). Similarly, for industrial and commercial load it was assumed that all the loads are industrial and commercial type respectively. In the 
practical situation, loads are not explicitly residential, industrial, and commercial; rather load class mix may be seen by distribution system depending on the nature of area being supplied. Therefore, a load class mix of residential, industrial and commercial load were also adopted. This is also indicated in Table A1 along with the load data.

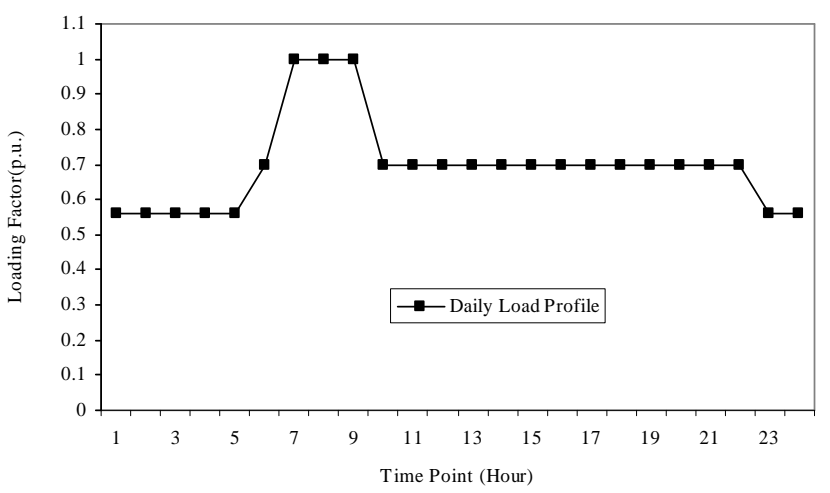

Fig. 1: Daily load Profile

Fig. 1 shows the daily load curve of different types of load models throughout a year for a distribution system. Time points presented, are representative of three loading conditions, which are low, medium and peak load, respectively. Over these time-varying load conditions, the DG is operated at the range of 0-5.50 p.u.. It is well known that daily load curve varies season to season. Due to complexity in load curve variation analysis is done with assumption that daily load curve is same in whole year.

\section{DATA PREPARATION}

After the above-mentioned test systems and cases to be studied are finalized, the power flow solution for the 16-bus standard distribution system is obtained in the following fashion. First of all a DG size is considered in a practical range (0-4.00 p.u). The DG of 0.0 p.u. corresponds to system without DG whereas 4.00 p.u corresponds to a case when all the real power requirements are met by DG. It is considered that the DG is operated at unity p.f. Each bus of 16-bus system is considered for the placement of a DG of given size from the range considered. Then load flow program is run for each of this case. The complete procedure to create data base is presented in flowchart given in fig. 2 .

The flowchart for the selection of minimum loss configuration, for selected load type from the database is provided in fig.3. A similar algorithm is used for minimum MVA and cost criterion also. For energy loss analysis Database is generated for three types of loading conditions: peak, medium and low loads. According to variation of load profile, annual system energy loss is calculated for minimum energy loss configuration. Flowchart for selection of minimum annual system energy loss for optimal size and location of distributed generator is same as fig.3. The bus voltages and MVA flow of lines occurring beyond the limits were treated as voltage and line violations respectively.

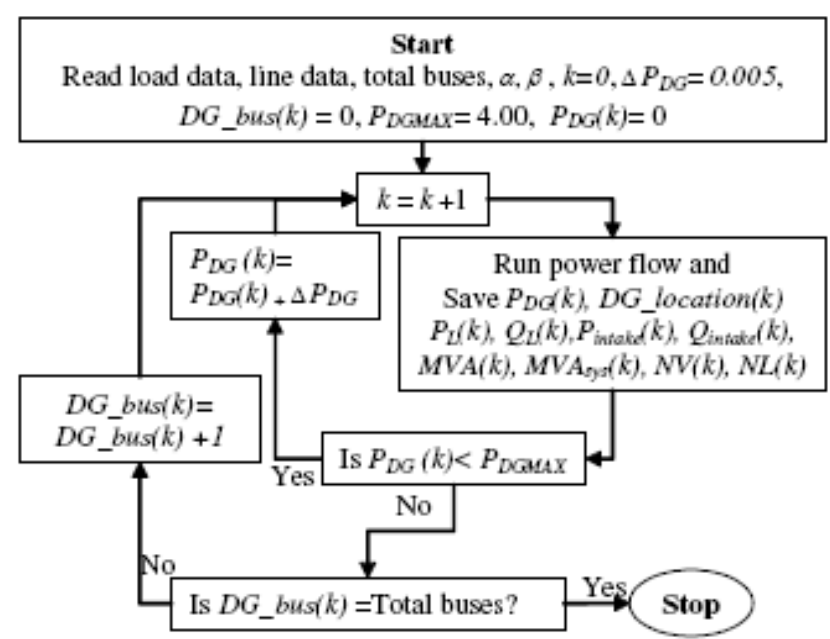

Fig.2. Flowchart for creation of database

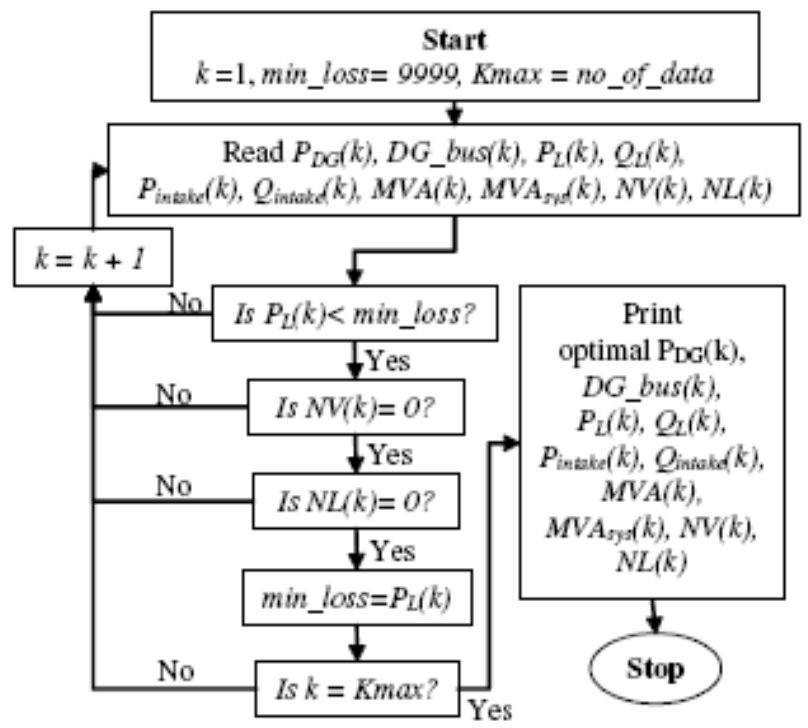

Fig.3. Flowchart for selection of minimum loss configuration from database

\section{DG PlanNing MODEL}

The mathematical formulation for DG planning problem from the prospective of the disco is presented in this section. In this problem, we consider the disco to be the sole owner and operator of the distribution network system that supplies electricity to its customers. The disco has the option to purchase the power from any genco (DG company) in the system through bilateral contracts and it also has the possibility to purchase power directly from a day-ahead electricity spot market. The purchased power exchange from the main grid to distribution network takes place at the junction substation. The disco is required to meet customers demand growth.

The problem of optimal size and placement of distributed generator in distribution system is analyzed with different criteria: Power loss, System MVA, System cost and System Energy loss. The objective functions for above criterion are stated as

Case-I: Power Loss Minimization 
Min. $\left[P_{L D G}\right]$

$$
P_{L D G}=\sum_{i=1}^{n}\left(P_{i}-P_{D G}\right)
$$

subject to

$$
P_{i}=\left|V_{i}\right| \sum\left|V_{k}\right|\left[g_{i k} \cos \left(\theta_{i}-\theta_{k}\right)+b_{i k} \sin \left(\theta_{i}-\theta_{k}\right)\right]
$$

(6)

$$
P_{\text {line }(i, j)} \leq P_{\text {line }(i, j) \max }
$$

In the above formulation $i$ is the location which ranges from bus 2 to $n$, bus 1 being the slack node or the feeder node and $\mathrm{n}$ being the total number of bused in the system. The variables $P_{i}, Q_{i}, V_{i}$, and $\theta_{\mathrm{i}}$ carry the usual meaning as in power flow studies. $P_{D i}$ and $Q_{D i}$ are the real and reactive load at bus $i$. The important operational constraints are addressed by equations (6) and (7).

\section{Case-II: MVA intake Minimization}

Min. $\left[\mathrm{MVA}_{\text {sys }}\right]$

The $M V A_{s y s}$ is the total MVA intake by the DISCO and is defined as

$M V A_{\text {sys }}=\left[\left(P_{\text {int } a k e}+P_{D G}\right)^{2}+\left(Q_{\text {int ake }}\right)^{2}\right]^{1 / 2}$

\section{Case-III: Cost function Minimization}

The problem addressed in the paper is a long-term planning with the objective of maximizing the disco profit (Profit=Revenue-Cost) implies maximizing the revenue (from the sell of electricity) and minimizing the cost (DG installation and operation).

In our problem, the disco has a committed total customer demand that is to be satisfied. Hence, the total revenue earned by the disco is proportional to the retail price it charges from its customers. In this analysis, we assume that the retail price is fixed. This is a reasonable assumption since these are often regulated by local authorities or the government, or there could even be a retail price cap. Hence, the problem of maximizing the disco profit reduces to the minimization of costs only.

The proposed objective function (9) aims to minimize the investment and operating costs of proposed DGs, payment toward purchased power by the disco, payments toward power loss compensation services. The mathematical formulation as described in (9) as follows:

$$
\operatorname{Min} \cdot\left[J_{\cos t}\right]
$$

$$
\begin{aligned}
& f_{\cos t}=T_{P} \cdot \sum_{i=1}^{n}\left(P_{S S}^{P}(i, V) \cdot \rho+P_{D G i}^{P} \cdot C_{c i}\right)+ \\
& +T_{M} \cdot \sum_{i=1}^{n}\left(P_{S S}^{M}(i, V) \cdot \rho+P_{D G i}^{M} \cdot C_{c i}\right)+ \\
& T_{L} \cdot \sum_{i=1}^{n}\left(P_{S S}^{L}(i, V) \cdot \rho+P_{D G i}^{L} \cdot C_{c i}\right)+\sum_{1=1}^{n} C_{f i} \cdot P_{D G i}^{C A P}
\end{aligned}
$$

where

$\mathrm{P}_{\mathrm{DGi}}^{\mathrm{CAP}}=\mathrm{DG}$ capacity limit at $\mathrm{i}^{\text {th }}$ bus(MW);

$\mathrm{P}_{\mathrm{SSi}}^{\mathrm{p}}=$ Power purchased by disco at $\mathrm{i}^{\text {th }}$ bus on peak loading Condition (MW);

$\mathrm{P}_{\mathrm{SSi}}^{\mathrm{M}}=$ Power purchased by disco at $\mathrm{i}^{\text {th }}$ bus on low loading Condition (MW);

$\mathrm{P}_{\mathrm{SSi}}^{\mathrm{L}}=$ Power purchased by disco at $\mathrm{i}^{\text {th }}$ bus on medium loading

$$
\text { Condition (MW); }
$$

$\mathrm{P}_{\mathrm{DGi}}^{\mathrm{P}}=$ Power generated by DG at $\mathrm{i}^{\text {th }}$ bus at peak loading Condition (MW);

$\mathrm{P}_{\mathrm{DGi}}^{\mathrm{M}}=$ Power generated by $\mathrm{DG}$ at $\mathrm{i}^{\text {th }}$ bus at medium loading Condition (MW);

$\mathrm{P}_{\mathrm{DGi}}^{\mathrm{L}}=$ Power generated by DG at $\mathrm{i}^{\text {th }}$ bus at low loading Condition (MW);

$\rho=$ Electricity Market Price $(\$ / M W-h r)$

$\mathrm{C}_{\mathrm{ci}}=$ Hourly DG operating cost at $\mathrm{i}^{\text {th }}$ bus ( $\$ / \mathrm{MW}-\mathrm{hr}$ )

$\mathrm{C}_{\mathrm{fi}}=$ Hourly DG investment cost (\$/MW-hr)

$T_{P}=$ Average duration of peak load condition;

$\mathrm{T}_{\mathrm{M}}=$ Average duration of medium load condition;

$\mathrm{T}_{\mathrm{L}}=$ Average duration of low load condition and

$\mathrm{V}=$ Bus voltage (Volt).

Different loading levels expected by the disco are modeled as shown in fig. 1.The objective function is the hourly cost (in $\$ / \mathrm{hr}$ ) and the total DG investment and operation costs are hourly costs.

The objective function (8) is minimized subject to various operating constraints to satisfy the electrical requirements for distribution network, constraints on DG operation, as well as constraints on investment resources. These constraints are discussed as follows:

1. Power-Conservation Limits: The algebraic sum of all incoming and outgoing power including line losses over whole distribution network and power generated from DG unit should be equal zero.

$$
\begin{aligned}
& P_{S S}^{X}(i, V)=\sum_{i=2}^{n}\left(P_{D}^{X}(i, V)+\sum_{n=1}^{N O L} P_{\text {loss }}(V)-P_{D G i}^{X}\right. \\
& X \forall(P \text { or } M \text { or } L) \\
& N O L=\text { no of lines, } P_{D}=\text { power demand }(M W)
\end{aligned}
$$


2. Distribution Line Capacity Limits: Power flow through any distribution feeder must comply with the thermal capacity of the line.

$P_{\text {line }(i, j)} \leq P_{\text {line }(i, j) \max }$

3. Voltage Drop Limits: The voltage drop limts depend on the voltage regulation limits provided by the disco.

$0 \leq\left|V_{i}-V_{j}\right| \leq \Delta V_{\text {line }(i, j) \max }$

4. DG Operation Limits: The power generated from the DG must be less than the DG capacity.

$P_{D G i}^{C A P} \geq \max \left[P_{D G i}^{P}, P_{D G i}^{M}, P_{D G i}^{L}\right]$

5. Investment Resources Limits: The disco often has to carry out investment planning decision making while considering its financial limits. This limit imposes a condition on DG planning capacity.

$$
\sum_{1=1}^{n} C_{f i} \cdot P_{D G i}^{C A P} \leq \text { financial Limit }
$$

\section{Case-IV : Energy Loss Minimization}

Min. $\left[\mathrm{J}_{\text {energy }}\right]$

Annual system energy loss

$$
\begin{aligned}
& J_{\cos t}=T_{P} \cdot \sum_{i=1}^{n} \rho \cdot P_{S S}^{P}(i, V)+ \\
& +T_{M} \cdot \sum_{i=1}^{n} \rho \cdot P_{S S}^{M}(i, V)+T_{L} \cdot \sum_{i=1}^{n} \rho \cdot P_{S S}^{L}(i, V)
\end{aligned}
$$

$$
\left(\mathrm{T}_{\mathrm{P}}+T_{\mathrm{M}}+T_{\mathrm{L}}\right) \leq T
$$

Where

T =Total duration of DG operation;

Other symbols have same meanings as mentioned in cost minimization analysis. Energy loss minimization analysis is done under operational constraints 1,2 and 3 as stated in cost minimization analysis.

\section{TEST CASES}

The following test cases are developed for DG planning scenario:

\section{Single DG Case:}

In the given load scenario customer decides to embed a single distributed generation resource of 0.8 p.u. on buses ( 7 and 14) in 16-bus system. The effect of load models on the following studies is to be made.

29) Number of voltage limit violation,

30) Number of line limit violations,

31) MVA, real and reactive p.u. demand on the main substation,

32) Loss reduction in real and reactive power, and

33) Saving of MVA capacity on the main substation.

\section{Multiple DG Case:}

In the given load scenario customers decide to embed three distributed generators of $0.20,0.30$ and 0.20 p.u. on set of buses $(7,10$ and 14) in 16-bus system. The effect of load models on the following studies is to be made.

1) Number of voltage limit violations,

2) Number of line limit violations,

3) MVA, real and reactive p.u. demand on the main substation,

4) Loss reduction in real and reactive power, and

5) Saving of MVA capacity on the main substation.

\section{Optimal DG location and size planning:}

In the given load scenario DISCO decides to embed a distributed generator at optimal location and of optimal size (without undergoing any system upgrades) for,

1) Loss reduction or

2) Reduction of the main substation MVA intake (system upgrade deferment).

3) Reduction of operational cost

4) Annual energy loss reduction

Effect of load models on the optimal location and size of distributed generators are studied.

\section{Effect of error in load modeling:}

In the given load scenario DISCO decides to embed a distributed generator at optimal location and of optimal size for

1) Loss reduction or

2) Reduction of the main substation MVA intake (System upgrade deferment).

Planning for optimal size and location for the two cases has been done assuming constant power load demand. The effect of load models (actual load type) on technical feasibility is studied.

To observe the effect of DG placement, various indices are discussed here. These are:

A. Real and reactive power loss indices (ILP and ILQ):

The real and reactive power loss indices are defined as

$$
\begin{aligned}
& I L P=\frac{\left[P_{L D G}\right]}{\left[P_{L}\right]} \times 100, \\
& I L Q=\frac{\left[Q_{L D G}\right]}{\left[Q_{L}\right]} \times 100
\end{aligned}
$$

Farther the values from one better is the benefits in terms of loss reduction accrued to DG location and size.

\section{B. Voltage profile Index (IVD):}

This index is related to the maximum voltage drop which, in this case, will consider the maximum drop between each node and the root node. This index could be also used to find prohibitive locations for DG considering pre-established voltage drop limits. In this way, farther the index to unity better is the network performance. The IVD can be defined as follows,

$$
I V D=100 \times \max _{i=2}^{n}\left(\frac{\left|\bar{V}_{1}\right|-\left|\bar{V}_{i}\right|}{\left|\bar{V}_{1}\right|}\right)
$$

The benefit of placing DG in a system in context of voltage profile improvements is measured by finding the difference in IVD between system with and without DG. 


\section{MVA capacity index(IC):}

As a consequence of supplying power near to loads, MVA flows may diminish in some sections of the network, thus releasing more capacity, but in other sections they may also increase to levels beyond distribution line limits. This index gives important information about the level of MVA flow/currents through the network regarding the maximum capacity of conductors. This gives the information about need of system line upgrades. Positive values (calculated MVA flow values lower than the MVA capacity) of the index give the amount of capacity released in term of line flows whereas, the negative values indicate otherwise.

$$
I C=100 \times \max _{i=1}^{m}\left(\frac{\left|\overline{S_{i j}}\right|}{\left|\overline{C S_{i j}}\right|}\right)
$$

The benefit of placing DG in a system in context of line capacity released is measured by finding the difference in IC between system with and without DG.

\section{SIMULATION RESULTS AND DISCUSSIONS}

The test study is carried out on 16-bus system. The line data, load data, line loading limits for the 16-bus system are described in Appendix I. The real $\left(P_{i}\right)$ and reactive $\left(Q_{i}\right)$ loads at buses corresponds to peak load. The medium loading condition is taken as $70 \%$ of the peak load at each bus, whereas, $80 \%$ of the medium loading condition is considered as low load.

Case 1: Single DG Case: Distributed generation resource $\left(P_{D G}\right)$ of 0.8 p.u. on bus 7.

The summary of results obtained in this case for different load models is depicted in Table 2. The various indices as discussed above are presented in Figs. 4, 5, and 6.

Constant Power Load Model: From Table 2 it is observed that there is decrease of 0.8107 p.u. in the real ( $\left.P_{\text {int ake }}\right)$, 0.0097 p.u. in reactive ( $Q_{\text {int ake }}$ ) and 0.6607 p.u. in MVA demands at the main substation due to placement of DG. Furthermore, real power loss $\left(P_{L}\right)$ and reactive power loss ( $Q_{L}$ ) also get reduced by 0.0107 p.u. and 0.0097 p.u. respectively. The overall system MVA $\left(M V A_{s y s}\right)$ of the DISCO is reduced by 0.0139 p.u.. However, there were no voltage as well as line limit violations for both the situations i.e. with and without DG.

Residential Load Model: For residential model, a similar trend of reduction with DG placement is observed for real $\left(P_{\text {int ake }}\right)$ and MVA demands, real power loss $\left(P_{L}\right)$ and reactive power loss $\left(Q_{L}\right)$ except for reactive demand ( $\left.Q_{\text {int ake }}\right)$. As opposed to the case of constant load model, in this case despite the addition of a DG, there is increase in reactive power intake from the main substation.

It is observed that the reduction in real power intake is 0.8007 p.u., in MVA 0.6274 p.u., in real power loss 0.0107 p.u. and in reactive power loss 0.0100 p.u.; whereas in case of constant load model, these values are 0.8107 p.u., 0.6607 p.u., 0.0107 p.u. and 0.0097 p.u. respectively. It is to be noted that all these reductions are significantly smaller than those of constant load model. As far as reactive power demand at the main substation is concerned, it is seen that in case of residential load model, placement of DG raises reactive demand ( by 0.0129 p.u.) whereas in case of constant load model, the reactive demand is decreased by 0.0097 p.u. which is almost of the same order.

Also there is no line limit violation in this case when DG is placed in the system as against the case of constant power load model. However, there were no voltage limit violations and line limit violation for the residential load model without DG.

Industrial Load Model: It can be observed that the trend of reduction in real, reactive and MVA demand with DG placement, as observed for the cases of constant and residential load model, is maintained in this case also. It is to be noted that all these reductions are significantly smaller than those of constant load model and marginally larger than those of residential load models. Further the increase in reactive power demand is distinctly higher than those observed for the case of residential as well as constant load model. There were no voltage as well as line limit violations for both the situations i.e. with and without DG.

Commercial Load Model: In this case it was observed that the reductions in all the quantities indicated in Table 2 except the reactive power demand, are having their lowest values compared to all other considered load models. It is further to be noted that the total real power demand of the system ( $P_{\text {int ake }}+P_{D G}=0.6727+0.8=1.4727$ p.u.) with DG is marginally higher than that for system without DG i.e. 1.4671 p.u. It is to be emphasized that despite the reduction in real and reactive power losses, the overall real and reactive power intake may in fact increase making void the advantage of loss reduction. This is due to improvement in voltage profile which results in an increase in loads that exceeds the amount of loss reduction. In such a case if the disco happens to pay same amount/MWh, it losses instead gaining from DG placement. This may not be detectable if proper load model is not taken into account. Also there is one line limit violation in this case when DG is placed in the system.

Mixed Load Model: The mixed load model corresponds to practical distribution system loading. The reduction in real and MVA power demand is less compared to constant load model. The reductions in real and reactive losses are also less when compared to constant load model.

TABLE 2

Summary of Results Obtained for Case 1: Single DG Case

\begin{tabular}{|l|l|l|l|l|l|l|l|l|}
\multicolumn{8}{|c|}{ Summary of Results Obtained for Case 1: Single DG Case } \\
\hline & $P_{\text {intake }}$ & $Q_{\text {intake }}$ & $S_{\text {intake }}$ & $M V A_{\text {sys }}$ & $P_{L}$ & $Q_{L}$ & $\begin{array}{l}N \\
V\end{array}$ & $N L$ \\
\hline Const. & & & & & & & & \\
& & & & & & & & \\
\hline WDG & 0.620 & 0.7043 & 0.938 & 1.5854 & 0.005 & 0.004 & 0 & 0 \\
& 4 & & 6 & & 4 & 3 & & \\
\hline WOD & 1.431 & 0.7140 & 1.599 & 1.5993 & 0.016 & 0.014 & 0 & 0 \\
G & 1 & & 3 & & 1 & 0 & & \\
\hline Diff. & $\mathbf{0 . 8 1 0}$ & $\mathbf{0 . 0 0 9 7}$ & $\mathbf{0 . 6 6 0}$ & $\mathbf{0 . 0 1 3 9}$ & $\mathbf{0 . 0 1 0}$ & $\mathbf{0 . 0 0 9}$ & & \\
& $\mathbf{7}$ & & $\mathbf{7}$ & & $\mathbf{7}$ & $\mathbf{7}$ & & \\
\hline Res. & & & & & & & & \\
& & & & & & & \\
\hline WDG & 0.652 & 0.7762 & 1.014 & 1.6468 & 0.006 & 0.004 & 0 & 0 \\
& 4 & & 0 & & 0 & 9 & & \\
\hline WOD & 1.453 & 0.7633 & 1.641 & 1.6414 & 0.016 & 0.014 & 0 & 0 \\
G & 1 & & 4 & & 7 & 9 & & \\
\hline Diff. & $\mathbf{0 . 8 0 0}$ & $\mathbf{- 0 . 0 1 2}$ & $\mathbf{0 . 6 2 7}$ & $\mathbf{- 0 . 0 0 5}$ & $\mathbf{0 . 0 1 0}$ & $\mathbf{0 . 0 1 0}$ & & \\
& $\mathbf{7}$ & $\mathbf{9}$ & $\mathbf{4}$ & $\mathbf{4}$ & $\mathbf{7}$ & $\mathbf{0}$ & & \\
\hline Ind. & & & & & & & & \\
\hline WDG & 0.637 & 0.8134 & 1.027 & 1.6516 & 0.006 & 0.005 & 0 & 0 \\
& 4 & & 3 & & 3 & 1 & \\
\hline WOD & 1.435 & 0.7883 & 1.638 & 1.6380 & 0.016 & 0.014 & 0 & 0 \\
G & 8 & & 0 & & 7 & 5 & \\
\hline
\end{tabular}




\begin{tabular}{|l|l|l|l|l|l|l|l|l|}
\hline Diff. & $\begin{array}{l}\mathbf{0 . 8 0 8} \\
\mathbf{4}\end{array}$ & $\begin{array}{l}\mathbf{- 0 . 0 2 5} \\
\mathbf{1}\end{array}$ & $\begin{array}{l}\mathbf{0 . 6 1 0} \\
\mathbf{7}\end{array}$ & $\begin{array}{l}\mathbf{- 0 . 0 1 3} \\
\mathbf{6}\end{array}$ & $\begin{array}{l}\mathbf{0 . 0 1 0} \\
\mathbf{4}\end{array}$ & $\begin{array}{l}\mathbf{0 . 0 0 9} \\
\mathbf{4}\end{array}$ & & \\
\hline Com. & & & & & & & & \\
& & & & & & & & \\
\hline WDG & 0.672 & 0.7642 & 1.018 & 1.6562 & 0.005 & 0.004 & 0 & 0 \\
& 7 & & 1 & & 9 & 9 & & \\
\hline WOD & 1.467 & 0.7553 & 1.650 & 1.6594 & 0.017 & 0.014 & 0 & 0 \\
G & 1 & & 1 & & 1 & 6 & & \\
\hline Diff. & $\mathbf{0 . 7 9 4}$ & $\mathbf{- 0 . 0 0 8}$ & $\mathbf{0 . 6 3 3}$ & $\mathbf{0 . 0 0 3 2}$ & $\mathbf{0 . 0 1 1}$ & $\mathbf{0 . 0 0 9}$ & & \\
& $\mathbf{4}$ & $\mathbf{9}$ & $\mathbf{0}$ & & $\mathbf{2}$ & $\mathbf{7}$ & & \\
\hline Mix. & & & & & & & & \\
& & & & & & & & \\
\hline WDG & 0.658 & 0.7752 & 1.017 & 1.6518 & 0.006 & 0.004 & 0 & 0 \\
& 6 & & 2 & & 1 & 8 & & \\
\hline WOD & 1.455 & 0.7641 & 1.644 & 1.6440 & 0.016 & 0.014 & 0 & 0 \\
G & 6 & & 0 & & 8 & 8 & & \\
\hline Diff. & $\mathbf{0 . 7 9 7}$ & $\mathbf{- 0 . 0 1 1}$ & $\mathbf{0 . 6 2 6}$ & $\mathbf{- 0 . 0 0 7}$ & $\mathbf{0 . 0 1 0}$ & $\mathbf{0 . 0 1 0}$ & & \\
& $\mathbf{0}$ & $\mathbf{1}$ & $\mathbf{8}$ & $\mathbf{8}$ & $\mathbf{7}$ & $\mathbf{0}$ & & \\
\hline
\end{tabular}

The increase in reactive power demand is also observed in practical case of mixed load as in case of other load model except for constant load model. The line limit violation is observed when a DG is placed in the system.

The real and reactive loss indices ILP and ILQ are depicted in Fig. 4 along with the corresponding values. It can be observed that the indices indicating the effect of DG placement are high in case of constant power load model assumption, whereas for non-constant power load model (actual system) these are lower. Hence planning studies for evaluating the advantage of DG placement based on constant power load model may indicate lower system losses.

The difference between the IVDs of system with and without DG is plotted in Fig. 5 indicating the improvement in voltage profile due to DG placement. In this case also, the constant power load model assumption gives a picture of higher improvement as compared to actual system (non-constant power load model).

The IC indices depicting the available MVA capacity in the system with and without DG for different load models are shown in Fig. 6. For the system without DG, the available capacity for different load models are not much different compared to the case when DG is place; in this case in an actual system, constant power load model assumption based studies gives false indication of high available MVA capacity, whereas the fact is other wise.

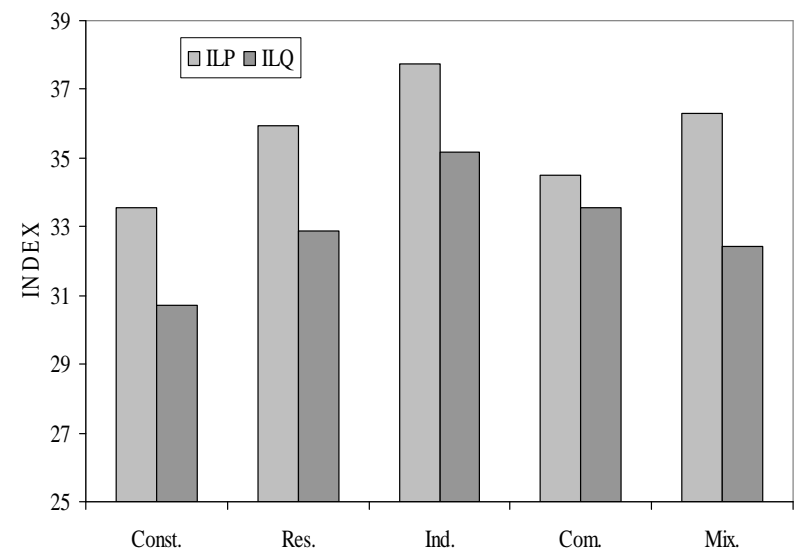

Fig. 4: ILP and ILQ for different load models (Case 1)

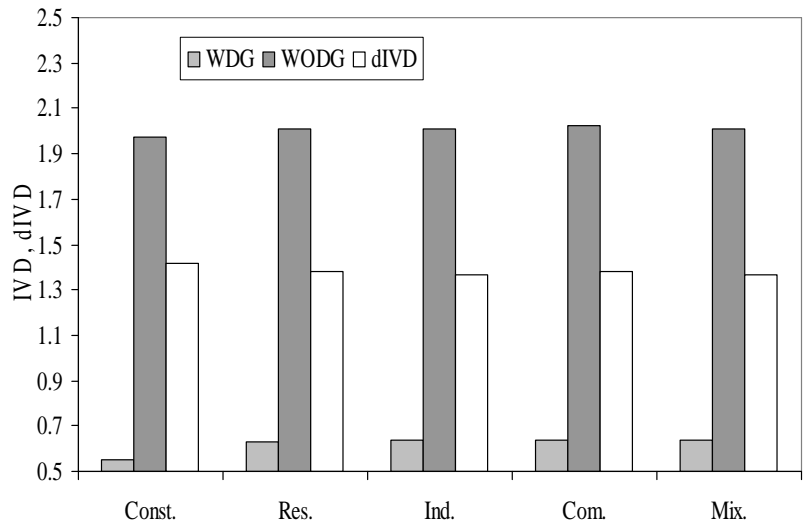

Fig. 5: IVD and dIVD for different load models (Case 1)

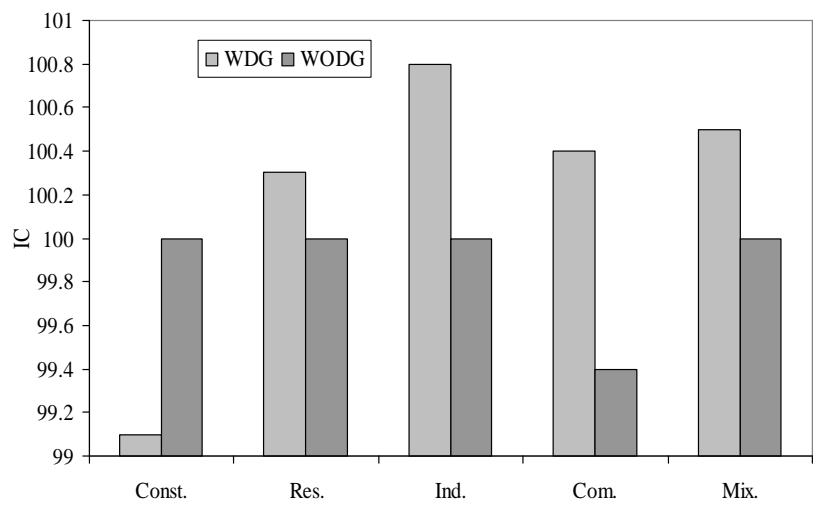

Fig. 6: IC for different load models (Case 1)

Case 2: Multiple DG Case:

Three distributed generators of $0.20,0.30$, and 0.20 p.u. ( $P_{D G}=0.7$ p.u.) on buses 7,10 , and 14 respectively. The summary of results obtained in this case for different load models is depicted in Table 3. The differences of all the quantities indicated above for the system with DG and without DG are also indicated for different load types.

Numbers of voltage and line limit violations have also been shown in the Table 3 for different load types with and with and without DG as well.

Constant Power Load Model: From Table 3, for constant load model, it is observed that there is decrease of 0.7110 p.u. in the real ( $\left.P_{\text {int ake }}\right), 0.0097$ p.u. in reactive $\left(Q_{\text {int ake }}\right)$ and 0.5920 p.u. in MVA demands at the main substation due to placement of DG. Furthermore, real power loss $\left(P_{L}\right)$ and reactive power loss $\left(Q_{L}\right)$ also get reduced by 0.0110 p.u. and 0.0097 p.u. respectively. The overall system MVA of the DISCO is reduced by 0.0142 p.u..

It can be concluded that when constant load model is assumed the reduction in real and reactive power losses is directly translated as reduction in overall system MVA $\left(M V A_{s y s}\right)$ and giving erroneous indications of higher benefits of DG placement.

This is because the effect of voltage profile on loads is not taken into account. However, there were no voltage as well as line limit violations for both the situations i.e. with and without DG.

Residential Load Model: For residential model a similar trend of reduction is observed for real $\left(P_{\text {int ake }}\right)$ and MVA demands, real power loss $\left(P_{L}\right)$ and reactive power loss $\left(Q_{L}\right)$ except for reactive demand ( $Q_{\text {int ake }}$ ) with DG placement. 
As opposed to the case of constant load model, and observed in case 1 , in this situation also despite the addition of a DG, there is increase in reactive power intake from the main substation.

It is to be noted that reductions, real power intake, system MVA, real, and reactive loss are significantly smaller than those of constant load model. As far as reactive power demand at the main substation is concerned, it is seen that in case of residential load model, placement of DG raises reactive demand (by 0.0075 p.u.) whereas in case of constant load model, the reactive demand (by 0.0097 p.u.) is decreased almost by same order. Also, unlike the constant power load assumption, the system real and reactive losses do not directly reflect into the overall system intake.
However, placement of DG does not violate voltage and line limits.

Industrial Load Model: It can be observed that the trend of reduction in real, reactive and MVA demand with DG placement, as observed for the cases of constant and residential load model, is maintained in this case also. However, the overall system MVA demand reduction is more than the residential load model but less than constant load model assumption. Further the increase in reactive power demand is almost doubled and is distinctly higher than those observed for the case of residential as well as constant load model. There were no voltage as well as line limit violations for both the situations i.e. with and without DG.

TABLE 3Summary of Results Obtained for Case 2: Multiple DG Case

\begin{tabular}{|c|c|c|c|c|c|c|c|c|}
\hline & $P_{\text {intake }}$ & $Q_{\text {intake }}$ & $S_{\text {intake }}$ & $M V A_{s y s}$ & $P_{L}$ & $Q_{L}$ & $N V$ & $N L$ \\
\hline \multicolumn{9}{|l|}{ Const. } \\
\hline WDG & 0.7201 & 0.7043 & 1.0073 & 1.5851 & 0.0051 & 0.0043 & 0 & 1 \\
\hline WODG & 1.4311 & 0.7140 & 1.5993 & 1.5993 & 0.0161 & 0.0140 & 0 & 0 \\
\hline Diff. & 0.7110 & 0.0097 & 0.5920 & 0.0142 & 0.0110 & 0.0097 & & \\
\hline \multicolumn{9}{|l|}{ Res. } \\
\hline WDG & 0.7499 & 0.7708 & 1.0754 & 1.6420 & 0.0057 & 0.0048 & 0 & 1 \\
\hline WODG & 1.4531 & 0.7633 & 1.6414 & 1.6414 & 0.0167 & 0.0149 & 0 & 0 \\
\hline Diff. & 0.7032 & -0.0075 & 0.5660 & -0.0006 & 0.0110 & 0.0101 & & \\
\hline \multicolumn{9}{|l|}{ Ind. } \\
\hline WDG & 0.7266 & 0.8051 & 1.0845 & 1.6381 & 0.0060 & 0.0051 & 0 & 1 \\
\hline WODG & 1.4358 & 0.7883 & 1.6380 & 1.6380 & 0.0167 & 0.0145 & 0 & 0 \\
\hline Diff. & 0.7092 & -0.0168 & 0.5535 & -0.0001 & 0.0107 & 0.0094 & & \\
\hline \multicolumn{9}{|l|}{ Com. } \\
\hline WDG & 0.7687 & 0.7599 & 1.0809 & 1.6536 & 0.0059 & 0.0051 & 0 & 1 \\
\hline WODG & 1.4671 & 0.7753 & 1.6501 & 1.6594 & 0.0171 & 0.0146 & 0 & 0 \\
\hline Diff. & 0.6984 & 0.0154 & 0.5692 & 0.0058 & 0.0112 & 0.0095 & & \\
\hline \multicolumn{9}{|l|}{ Mix. } \\
\hline WDG & 0.7550 & 0.7705 & 1.0787 & 1.6464 & 0.0058 & 0.0051 & 0 & 1 \\
\hline WODG & 1.4556 & 0.7641 & 1.6440 & 1.6440 & 0.0168 & 0.0148 & 0 & 0 \\
\hline Diff. & 0.7006 & -0.0064 & 0.5653 & -0.0024 & 0.0110 & 0.0097 & & \\
\hline
\end{tabular}

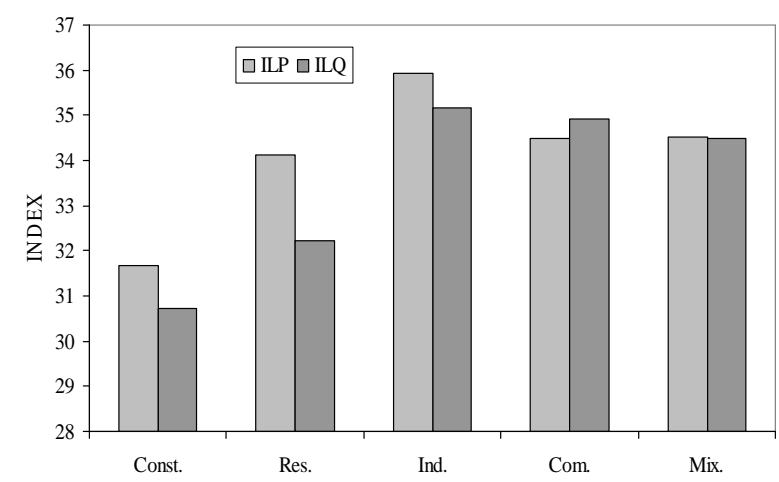

Fig. 7: ILP and ILQ for different load models (Case 2)

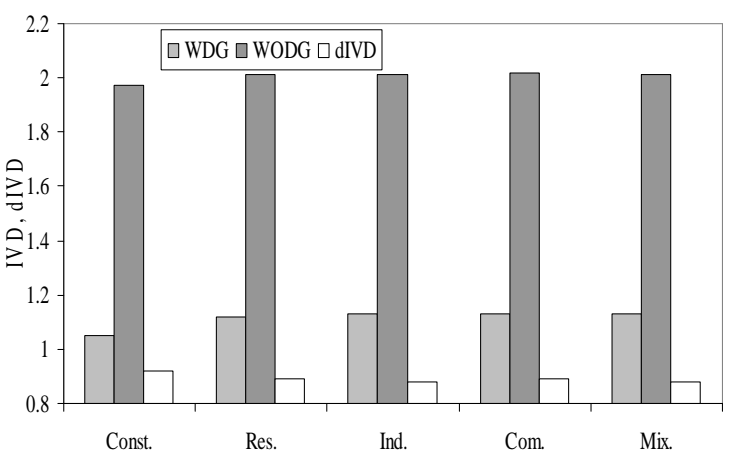

Fig. 8: IVD and dIVD for different load models (Case 2) 


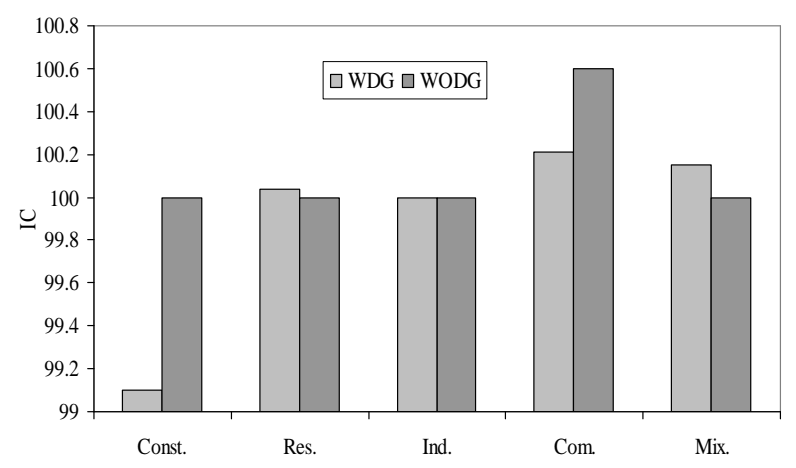

Fig. 9: IC for different load models (Case 2)

Commercial Load Model: In this case it was observed that the reductions in all the quantities indicated in Table 3 except the reactive power demand, are having their lowest values compared to all other considered load models. Further total real power demand of the system $\left(P_{\text {int ake }}+P_{D G}=0.7687+0.7=1.4687\right.$ p.u. $)$ with $\mathrm{DG}$ almost same to that of system without DG i.e. 1.4671 p.u.. The overall system MVA demand has increased marginally -0.0058 which is not observed in previously studied (constant, residential and industrial) load models for multiple DG case. In this load model also there were no voltage and line limit violations.

Mixed Load Model: In this practical load model the reduction in real and MVA demand power demand is less compared to constant load model. The reductions in real and reactive losses are also less when compared to constant load model. The increase in reactive power demand is also observed in practical case of mixed load as in case of other load model except for constant load model. No voltage limits and line limit violations were observed when a DG is placed in the system.

The real and reactive loss indices ILP and ILQ are depicted in Fig. 7 along with the corresponding values. Similar to the single DG case, the indices indicating the effect of DG placement are high in case of constant power load model assumption, whereas for non-constant power load model (except for the industrial load model) these are normally lower. The load models significantly affect the studies for evaluating the advantage of DG placement.

The difference between the IVDs of system with and without DG is plotted in Fig. 8 indicating the improvement in voltage profile due to DG placement. Similar to the single DG case, the constant power load model assumption gives a picture of higher improvement as compared to actual system (non-constant power load model).

The IC indices depicting the available MVA capacity in the system with and without DG for different load models are shown in Fig. 9. For the system without DG, the available capacity for different load models are not much different compared to the case when DG is placed; however unlike single DG case, constant power load model assumption based studies gives false indication of low available MVA capacity while the fact is otherwise?

\section{Case 3: Optimal DG Location and Size Planning}

The summary of results obtained for optimal DG size $\left(P_{D G}\right)$ and location for minimum loss configuration is given in Table 4 and for optimal DG location and size for minimum MVA intake from the main substation is given in Table 5. For different load models, the optimal DG Value which can be placed without upgrading the system $P_{L}, Q_{L}$, $P_{\text {intake }}, Q_{\text {intake }}$, MVA intake from the main substation, and $M V A_{\text {sys }}$ are also indicated in the Tables 4 and 5.

Constant Power load Model: From Table 4, for minimum loss configuration optimal size of DG which can be placed without need for upgrading the actual system is 0.77 p.u. at bus 8 .

TABLE 4

Minimum Loss Configuration for Different Load Models for Case 3: Optimal Location and Size planning

\begin{tabular}{|c|c|c|c|c|c|}
\hline & Const. & Res. & Ind. & Com. & Mix. \\
\hline $\begin{array}{l}\text { Optimal } \\
\text { Location }\end{array}$ & 8 & 8 & 7 & 7 & 7 \\
\hline DG Value & 0.770 & 0.690 & 0.775 & 0.765 & 0.820 \\
\hline $\begin{array}{l}P_{L} \\
\text { (Optimal) }\end{array}$ & 0.0054 & 0.0058 & 0.0062 & 0.0057 & 0.0058 \\
\hline$Q_{L}$ & 0.0043 & 0.0048 & 0.0050 & 0.0048 & 0.0047 \\
\hline$P_{\text {int ake }}$ & 0.6504 & 0.7617 & 0.6523 & 0.7070 & 0.6389 \\
\hline$Q_{\text {int } a k e}$ & 0.7043 & 0.7744 & 0.8123 & 0.7634 & 0.7757 \\
\hline $\begin{array}{l}P_{\text {int } a k e} \\
+P_{D G}\end{array}$ & 1.4204 & 1.4517 & 1.4273 & 1.4720 & 1.4589 \\
\hline $\begin{array}{l}\text { MVA } \\
\text { Intake }\end{array}$ & 0.9587 & 1.0862 & 1.0417 & 1.0404 & 1.0049 \\
\hline$M V A_{s y s}$ & 1.5854 & 1.6454 & 1.6423 & 1.6582 & 1.6523 \\
\hline \multicolumn{6}{|c|}{$\begin{array}{l}\text { Const.= Constant power load model, Res. }=\text { Residential load model, Ind.= } \\
\text { Industrial load model, Com.= Commercial load model, Mix. = Mixed load } \\
\text { model }\end{array}$} \\
\hline
\end{tabular}

The losses are reduced to 0.0054 when DG is placed optimally as compared to that without DG (0.0161 p.u., Table 3). The $Q_{L}, P_{\text {int ake }}, Q_{\text {int ake }}$, MVA, and $M V A_{\text {sys }}$ have also decreased consistent with constant power load model assumption.

From Table 5 it is seen that the location of DG for minimum MVA intake (to defer substation upgrades) is different (bus 3 ) as for the case of minimum loss criterion, of course, with different optimal DG size (1.430 p.u.). It also establishes a fact that for a constant power load model the reduced real and reactive power loss is translated into reduced MVA injections and therefore the change in location may be minimal when optimality criterion is changed from minimum loss to minimum MVA.

Residential Load Model: For residential model a similar trend of reduction is observed for real ( $\left.P_{\text {int ake }}\right)$ and MVA demands, real power loss $\left(P_{L}\right)$ and reactive power loss $\left(Q_{L}\right)$ except for reactive demand ( $Q_{\text {int ake }}$ ) with DG placement. As opposed to the case of constant load model, and observed in case 1 , in this situation also despite the addition of a DG, there is increase in reactive power intake from the main substation.

It is to be noted that reductions, real power intake, system MVA, real, and reactive loss are significantly smaller than those of constant load model. As far as reactive power demand at the main substation is concerned, it is seen that in case of residential load model, placement of DG raises reactive demand (by 0.0111 p.u.) whereas in case of constant load model, the reactive demand ( by 0.0097 p.u.) is decreased almost by same order. Also, unlike the constant power load assumption, the system real and reactive losses do not directly reflect into the overall system intake. 
However, placement of DG does not violate voltage and line limits.

Industrial Load Model: For industrial load model, minimum loss configuration requires optimal size of DG to be 0.775 p.u. on bus 7 . It can be observed that the size and location are significantly different than that for constant power and residential load models. The minimum real power loss which can be achieved by placing DG optimally is 0.0062 p.u. as compared to 0.0054 p.u. for constant power load model. Also the system $P_{\text {intake }}$ is marginally higher than that of constant power load model.

For the case of minimum MVA based optimal size and location, it is observed from Table 5 that optimal location of DG it is similar to that of residential load but quite different than that for constant power load model assumption. Similarly, optimal size of DG in case of industrial load model is close to that of residential load model and is significantly different than that of constant load model.

Commercial and Mixed Load Models: For these load models, minimum loss configuration requires optimal size of DG to be 0.765 p.u. (Commercial Load Model) and 0.820 p.u. (Mixed Load Model) on bus 7. It is remarkable to note that the DG size is significantly different from the case of load models considered so far $(0.690$ p.u. and 0.775 p.u. respectively for case of residential and industrial load models). The location is having the difference of 1 bus and same respectively compared to that of residential and industrial load models, though it is farthest from the root bus compared to the other load models. The minimum real power loss which can be achieved by placing DG is 0.0057 p.u. (Commercial Load Model) and 0.0058 p.u. (Mixed Load Model) which is significantly higher compared to constant load model. The real power intakes $(0.7070$ p.u. and 0.6389 p.u., for Commercial and Mixed Load Models respectively are significantly higher than that of constant power load model. The MVA intakes (1.0404 p.u. and 1.0049 p.u. for commercial and mixed load models respectively) are significantly higher than that of constant power load model.

For these load models, minimum MVA configuration requires optimal size of DG to be 1.465 p.u. ( Commercial Load Model ) and 1.455 p.u. (Mixed Load Model) on bus 2 . It is notable that the DG locations are entirely different for minimum loss and minimum MVA criteria for non-constant power load models.

It is important to note that the minimum MVA intake criterion gives same bus location (bus 2) for DG in case of non-constant power load models. This is explained as follows. If we take constant power load model assuming that the total system demand is $P_{D}$ and $Q_{D}$ for real and reactive power respectively. Let the real and reactive system losses without DG be $P_{L}$ and $Q_{L}$ respectively. The total MVA real and reactive demand from the main substation can be given by

$\begin{array}{ccccc}P & = & P_{D} & + & P_{L} \\ Q & & (21) & & \\ Q_{D} & & + & Q_{L}\end{array}$

Now if DG is placed in the system, let the real and reactive losses be $P_{L D G}\left(P_{L D G}<P_{L}\right)$ and $Q_{L D G}\left(Q_{L D G}\right.$ $<Q_{L}$ ) respectively, assuming that the DG placement is reducing the losses. The total real and reactive injection at the main substation is given by

$$
\begin{array}{lllll}
P & = & P_{D} & + & P_{L D G} \\
Q & = & Q_{D}{ }^{(23)} & + & Q_{L D G}
\end{array}
$$

Since constant power load assumption makes $P_{D}$ and $Q_{D}$ insensitive to DG placement, for $P$ and $Q$ to be minimum, it minimizes $P_{L D G}$ and $Q_{L D G}$, which is same as minimum loss criterion. However, in the case of non-constant power load models, apart from $P_{L D G}$ and $Q_{L D G}, P_{D}$ and $Q_{D}$ also become sensitive to DG placement. It is observed in such cases that the line limit constraints become more important than the loss because giving a voltage dependent representation to loads and to losses as in equations (1) and (2), the values of losses are negligible or very small as compared to $P_{D}$ and $Q_{D}$. Thus when non-constant load models are used, the DG location in general are near to the main substation where the line limits are maximum compared to other lines of the distribution system operated in radial configuration.

TABLE 5 Minimum MVA Configuration for Different Load Models for Case 3: Optimal Location and Size planning

\begin{tabular}{|l|l|l|l|l|l|}
\hline & Const. & Res. & Ind. & Com. & Mix. \\
\hline $\begin{array}{l}\text { Optimal } \\
\text { Location }\end{array}$ & 3 & 2 & 2 & 2 & 2 \\
\hline DG Value & 1.430 & 1.455 & 1.435 & 1.465 & 1.455 \\
\hline$P_{L}$ & 0.0133 & 0.0156 & 0.0156 & 0.0160 & 0.0159 \\
\hline$Q_{L}$ & 0.0118 & 0.0139 & 0.0139 & 0.0141 & 0.0142 \\
\hline$P_{\text {int } a k e}$ & -0.0017 & -0.0020 & 0.0001 & 0.0027 & 0.0007 \\
\hline$Q_{\text {int } a k e}$ & 0.7118 & 0.7651 & 0.7913 & 0.7567 & 0.7659 \\
\hline $\begin{array}{l}P_{\text {int } a k e} \\
+P_{D G}\end{array}$ & 1.4283 & 1.4530 & 1.4351 & 1.4677 & 1.4557 \\
\hline $\begin{array}{l}\text { MVA } \\
\text { Intake } \\
(\text { Optimal })\end{array}$ & 0.7118 & 0.7651 & 0.7913 & 0.7567 & 0.7659 \\
\hline \begin{tabular}{l}
$M V A_{\text {sys }}$ \\
\hline
\end{tabular} & 1.6321 & 1.6421 & 1.6388 & 1.6513 & 1.6449 \\
\hline $\begin{array}{l}\text { Const. = Constant power load model, Res. = Residential load model, Ind. } \\
=\text { Industrial load model, Com. = Commercial load model, Mix. = Mixed } \\
\text { load model }\end{array}$ & & & & \\
\hline
\end{tabular}

In order to minimize the MVA intake maintaining the line limits, network finds it suitable to place the DG at that node where it come across line of maximum MVA limits i.e. the line from main substation to the next node i.e. bus 2 . Hence in all the non-constant power load models, the optimum location comes out to be bus 2 when MVA intake is considered as objective function to be minimized. Since in this study the bus 1 was not considered to be a candidate location, the next bus, the bus 2, was selected.

Cost based Analysis: The problem of operational cost minimization addressed is a long-term planning with the objective of maximizing the disco profit (Profit=Revenue-Cost) implies maximizing the revenue (from the sell of electricity) and minimizing the cost (DG installation and operation).

In the problem, the disco has a committed total customer demand that is to be satisfied. Hence, the total revenue earned by the disco is proportional to the retail price it 
charges from its customers. In this analysis, we assume that the retail price is fixed. This is a reasonable assumption since these are often regulated by local authorities or the government, or there could even be a retail price cap. Hence, the problem of maximizing the disco profit reduces to the operational and investment costs only.

Cost analysis is done to examine the cost-effectiveness and feasibility of DG planning and find out the size and location of DG. A price of $70 \$ / \mathrm{MWh}$ is assumed as the base-case electricity market price. The natural gas fired generator set type DG is assumed for study. The fixed cost of such unit is $0.5 \mathrm{M} \$ / \mathrm{MW}$. The presently worth of the hourly DG fixed cost, considering a life-time of 30 years with a yearly payment of $0.05 \mathrm{M} \$$ and $9.15 \%$ discount rate, is calculated to be $137 \$ / \mathrm{MW}$-day. Assuming a gas price of $0.14 \$ /$ liter, the operating cost of the DG unit is calculated to be 42 \$/MWh [2].

Above loads are simulated with three types of loading conditions: Peak load (full load); Medium load (i.e. $70 \%$ of peak load) and Low load (i.e. $80 \%$ of medium load). Occurrence of above loading conditions is random throughout the day. It is assumed that loading condition changes according to daily load profile shown in fig. 1 .

Cost minimization analysis is performed with assumption that DG unit may operate in variable generation capacity and it may change as assessed DG's power generation schedule depending on load profile. This flexibility in power generation may reduce operational cost of the system. For the case of minimum cost based penetration of DG, It is observed from Table 6 that optimal location of DG with load models is same except constant load model. The lowest optimal value of cost function is observed in constant load model. This value is almost same for all load models.

From the results given in Table 6 it is also concluded that penetration of DG is not required during low loading period. The maximum optimal value is due to penetration of small generation capacity of DG during medium and peak loading periods for commercial and mixed load models.

It also promotes variable generation planning of DG. Almost full generation capacity (maximum demand) is utilized in constant, residential and industrial load models for time-varying loads during medium and peak loading period. It will be cost effective due to 0.0 p.u. operational cost during low load period. System real power losses for constant load model are $0.0050 / 0.0064 / 0.0133$ p.u. at low, medium and peak loading period respectively.

System real losses are almost same for all load models during low load period but for residential, commercial and mix load models it is $25 \%$ higher than constant and industrial load models i.e. $0.0077 / 0.0159$ p.u. at medium and peak loading period respectively. Similar effect on reactive power losses are also by DG placement.

TABLE 6 Minimum Cost Configuration for Different Load Models for Case 3: Optimal Location and Size planning

\begin{tabular}{|l|l|l|l|l|l|}
\hline \multirow{2}{*}{ Optimal Location } & Const. & Res. & Ind. & Com. & Mix. \\
\cline { 2 - 6 } & 3 & 2 & 3 & 2 & 2 \\
\hline $\begin{array}{l}\text { DG Val(Low } \\
\text { Loading) p.u. }\end{array}$ & 0 & 0 & 0 & 0 & 0 \\
\hline $\begin{array}{l}\text { DG Val(Med. } \\
\text { Loading) p.u. }\end{array}$ & 1.4 & 1.45 & 1.4 & 1.45 & 1.45 \\
\hline $\begin{array}{l}\text { DG Val(Peak } \\
\text { Loading) p.u. }\end{array}$ & 1.4 & 1.45 & 1.4 & 1.45 & 1.45 \\
\hline
\end{tabular}

\begin{tabular}{|c|c|c|c|c|c|}
\hline $\mathrm{P}_{\mathrm{LDG}}{ }^{\mathrm{L}}$ & 0.0050 & 0.0053 & 0.0049 & 0.0054 & 0.0051 \\
\hline $\mathrm{P}_{\mathrm{LDG}}{ }^{\mathrm{M}}$ & 0.0064 & 0.0075 & 0.0069 & 0.0078 & 0.0077 \\
\hline $\mathrm{P}_{\mathrm{LDG}}^{\mathrm{P}}$ & 0.0133 & 0.0156 & 0.0137 & 0.0159 & 0.0159 \\
\hline $\mathrm{Q}_{\mathrm{LDG}}^{\mathrm{L}}$ & 0.0043 & 0.0045 & 0.0046 & 0.0045 & 0.0045 \\
\hline $\mathrm{Q}_{\mathrm{LDG}}{ }^{\mathrm{M}}$ & 0.0057 & 0.0070 & 0.0061 & 0.0071 & 0.0070 \\
\hline $\mathrm{Q}_{\mathrm{LDG}}^{\mathrm{P}}{ }^{\mathrm{P}}$ & 0.0118 & 0.0140 & 0.0123 & 0.0141 & 0.0142 \\
\hline $\mathrm{P}_{\mathrm{SS}}^{\mathrm{L}}$ & 0.79742 & 0.8140 & 0.8008 & 0.8246 & 0.8168 \\
\hline $\mathrm{P}_{\mathrm{SS}}{ }^{\mathrm{M}}$ & 0.0169 & 0.0023 & 0.0212 & 0.0148 & 0.0054 \\
\hline $\mathrm{P}_{\mathrm{SS}}^{\mathrm{P}}$ & 0.0283 & 0.0030 & 0.0335 & 0.0176 & 0.0057 \\
\hline $\mathrm{Qss}_{\mathrm{L}}^{\mathrm{L}}$ & 0.3963 & 0.4335 & 0.4527 & 0.4275 & 0.4334 \\
\hline $\mathrm{Qss}^{\mathrm{M}}$ & 0.4957 & 0.5404 & 0.5648 & 0.5332 & 0.5404 \\
\hline Qss $^{P}$ & 0.7118 & 0.7651 & 0.7954 & 0.7567 & 0.7659 \\
\hline Cost_func (Optimal) & 1608.0 & 1639.7 & 1615.0 & 1660.2 & 1644.8 \\
\hline $\mathrm{MVA}_{\text {intake }}{ }^{\mathrm{L}}$ & 0.8904 & 0.9222 & 0.9199 & 0.9288 & 0.9247 \\
\hline $\mathrm{MVA}_{\text {intake }}{ }^{\mathrm{M}}$ & 0.4960 & 0.5404 & 0.5652 & 0.5334 & 0.5404 \\
\hline $\mathrm{MVA}_{\text {intake }}{ }^{\mathrm{P}}$ & 0.7124 & 0.7651 & 0.7961 & 0.7569 & 0.7659 \\
\hline
\end{tabular}

Real power demand is also affected by DG placement. Real power demand is maximum $(0.8246 / 0.0148 / 0.0176$ p.u. $)$ for commercial load model at low, medium and peak loading period in DG planning. Real power demand for mixed load model is almost same as commercial load model. Real power demand is almost same for residential and industrial load models in given loading profile. The reactive power demand for commercial load model is lowest $(0.4275 / 0.5332 / 0.7567$ p.u.) in comparison to other load models and it is maximum for industrial load model. MVA intake for industrial load model is 2.5209/1.6318/2.1902 p.u. (lowest) at low, medium and peak loading period respectively. It is maximum (2.5274/1.9573/2.7198 p.u.) for commercial load model.

If DG planning is done with commercial and mixed load models, full DG capacity is not utilized which is not economical. It is also observed from Table 6 that optimal location of DG is same to all load models except constant load model but for commercial and mixed load models same size of DG at $60 \%$ capacity is suitable for distributed generation planning.

Energy loss Analysis: Energy loss analysis for optimal size and location is performed with time-varying load models which are experienced in actual power system. Above loads are simulated with three types of loading conditions (Peak load; Medium load: $70 \%$ of peak load and Low load: $80 \%$ of medium load). Occurrence of above loading conditions is random throughout the day. It is assumed that loading condition changes according to daily load profile throughout the year shown in fig. 1.

It is observed from Table 7 that optimal location of DG with load models is same except constant ad industrial load models. The optimal value of annual energy loss is observed in residential load model. It is highest for constant and industrial load models. It also promotes variable generation planning of DG. Almost same generation capacity (maximum demand) is utilized in all load models for time-varying loads in whole day.

System real power losses for constant load model are 0.0041/0.0064/0.0133 p.u. at low, medium and peak loading period respectively. System real losses are almost same for 
industrial load model but for residential, commercial and mix load models it is $20 \%$ higher than constant load model i.e. $0.0051 / 0.0075 / 0.0156$ p.u. at low, medium and peak loading period respectively. Similar effect on reactive power losses are also by DG placement. Real power demand is also affected by DG placement. Real power demand is maximum (0.0335 p.u.) for industrial load model during DG planning.

Real power demand for residential load model is almost same as industrial load model. It is due to penetration of same generation capacity of DG for residential and industrial load models. Real power demand is lowest (0.0057 p.u.) for mix load model during peak load period. This deduction in real power demand is due to presence of mix load in system. The effect on reactive power demand is opposite to real power demand. MVA intake for constant load model is $0.3958 / 0.4960 / 0.7124$ p.u. (lowest) at low, medium and peak loading period respectively. It is maximum (0.4523/0.5652/0.7961 p.u.) for industrial load model.

\section{Case 4: Error in Load Model}

The common error of assuming constant load model instead of actual ones in DG planning is investigated in this case. This part of the work shows that DG location and size for a particular loading scenario will not reflect the planned benefits when put into actual system if constant power load model has been assumed in the planning studies.

In the previous case study as we have seen that the optimum location and size for minimum loss were bus 8 and 0.77 p.u. respectively. However, if the feeder loads are residential, industrial, commercial or mixed load type such a location will be technically infeasible for the planned load scenario.

It is depicted in Table 8 when DG at said location and size put into operation in an actual load model scenario will lead to line violations. This holds for all the non-constant power load models.

Also it can be observed from Table 8 that in fact the real and reactive losses are lower than those obtained during planning study. This may indicate that the constant power load model gives conservative estimate as far as losses are concerned. However, more important is the fact that actual real and reactive power intake from the main substation increases. This nullifies the advantage of loss reduction. Further getting conservative estimate of system losses for actual scenario obtained assuming constant power load model is also rendered meaningless. Compared to the case of planned scenario (constant power load model) in case of non-constant load models, MVA intake, System real power intake, and overall system MVA intake increase which is due to increased real and reactive power intake.

TABLE 7 Minimum Energy Loss Configuration for Different Load Models for Case 3: Optimal Location and Size planning

\begin{tabular}{|l|l|l|l|l|l|}
\hline \multirow{2}{*}{ Optimal Location } & Const. & Res. & Ind. & Com. & Mix. \\
\cline { 2 - 6 } & 3 & 2 & 3 & 2 & 2 \\
\hline $\begin{array}{l}\text { DG Val(Low } \\
\text { Loading) p.u. }\end{array}$ & 1.4 & 1.45 & 1.4 & 1.45 & 1.45 \\
\hline $\begin{array}{l}\text { DG Val(Med. } \\
\text { Loading) p.u. }\end{array}$ & 1.4 & 1.45 & 1.4 & 1.45 & 1.45 \\
\hline $\begin{array}{l}\text { DG Val(Peak } \\
\text { Loading) p.u. }\end{array}$ & 1.4 & 1.45 & 1.4 & 1.45 & 1.45 \\
\hline P $_{\text {LDG }}{ }^{\mathrm{N}}$ & 0.0041 & 0.0051 & 0.0042 & 0.0052 & 0.0049 \\
\hline $\mathrm{P}_{\text {LDG }}{ }^{\mathrm{M}}$ & 0.0064 & 0.0075 & 0.0069 & 0.0078 & 0.0077 \\
\hline $\mathrm{P}_{\text {LDG }}{ }^{\mathrm{P}}$ & 0.0133 & 0.0156 & 0.0137 & 0.0159 & 0.0159 \\
\hline
\end{tabular}

\begin{tabular}{|c|c|c|c|c|c|}
\hline $\mathrm{Q}_{\text {LDG }}{ }^{\mathrm{L}}$ & 0.0036 & 0.0046 & 0.0038 & 0.0046 & 0.0046 \\
\hline $\mathrm{Q}_{\mathrm{LDG}}{ }^{\mathrm{M}}$ & 0.0057 & 0.0070 & 0.0061 & 0.0071 & 0.0070 \\
\hline $\mathrm{Q}_{\mathrm{LDG}}^{\mathrm{P}}$ & 0.0118 & 0.0140 & 0.0123 & 0.0141 & 0.0142 \\
\hline $\mathrm{P}_{\mathrm{SS}}^{\mathrm{L}}$ & 0.0125 & 0.0019 & 0.0161 & 0.0128 & 0.0049 \\
\hline $\mathrm{P}_{\mathrm{SS}}{ }^{\mathrm{M}}$ & 0.0169 & 0.0023 & 0.0212 & 0.0148 & 0.0054 \\
\hline $\mathrm{P}_{\mathrm{SS}}{ }^{\mathrm{P}}$ & 0.0283 & 0.0030 & 0.0335 & 0.0176 & 0.0057 \\
\hline $\mathrm{Qss}^{\mathrm{L}}$ & 0.3956 & 0.4341 & 0.4550 & 0.4279 & 0.4340 \\
\hline $\mathrm{Qss}^{\mathrm{M}}$ & 0.4957 & 0.5404 & 0.5648 & 0.5332 & 0.5404 \\
\hline $\mathrm{Qss}^{\mathrm{P}}$ & 0.7118 & 0.7651 & 0.7954 & 0.7567 & 0.7659 \\
\hline $\begin{array}{l}\text { Annual_energy_func } \\
\text { (Optimal) }\end{array}$ & 146.73 & 19.783 & 183.522 & 127.312 & 46.428 \\
\hline $\mathrm{MVA}_{\text {intake }}{ }^{\mathrm{L}}$ & 0.3958 & 0.4341 & 0.4523 & 0.4281 & 0.4340 \\
\hline $\mathrm{MVA}_{\text {intake }}{ }^{\mathrm{M}}$ & 0.4960 & 0.5404 & 0.5652 & 0.5334 & 0.5404 \\
\hline $\mathrm{MVA}_{\text {intake }}{ }^{\mathrm{P}}$ & 0.7124 & 0.7651 & 0.7961 & 0.7569 & 0.7659 \\
\hline
\end{tabular}

Const. $=$ Constant power load model, Res. $=$ Residential load model, Ind.= Industrial load model, Com. $=$ Commercial load model, Mix. $=$ Mixed load model

Considering the optimal solution of DG placement for minimum MVA intake (DG size of 1.43 p.u. at bus 3 ) obtained using constant power load model assumption and implementing it on actual system which exhibits non-constant power load behavior, the results obtained can be summarized in Table 9.

From Table 9 it is observed that in this case also this location and size will not be suitable as far as line limits are concerned. Also the optimal MVA intake from the main substation for non-constant load models is higher than that obtained using constant load model. The other values of losses and system intakes are also indicated in the Table 9. In the case of DG placement based on minimum MVA intake criterion also, implementation of solution obtained using constant power load model on network exhibiting non-constant power load behavior, in general reduces the assumed benefits accrued to DG placement.

TABLE 8 Actual Load Model Scenario for Minimum Loss Configuration (DG of 0.77 p.u. at Bus 8) for Case 4: Error in Load Models

\begin{tabular}{|c|c|c|c|c|c|}
\hline & Const. & Res. & Ind. & Com. & Mix. \\
\hline$P_{L}$ & 0.0054 & 0.0062 & 0.0065 & 0.0058 & 0.0060 \\
\hline$Q_{L}$ & 0.0043 & 0.0048 & 0.0052 & 0.0049 & 0.0049 \\
\hline$P_{\text {intake }}$ & 0.6504 & 0.6828 & 0.6576 & 0.7033 & 0.6891 \\
\hline$Q_{\text {intake }}$ & 0.7043 & 0.7768 & 0.8144 & 0.7648 & 0.7758 \\
\hline$P_{\text {intake }}+P_{D G}$ & 1.4204 & 1.4528 & 1.4276 & 1.4733 & 1.4591 \\
\hline$S_{\text {intake }}$ & 0.9587 & 1.0342 & 1.0467 & 1.0390 & 1.0377 \\
\hline$M V A_{\text {sys }}$ & 1.5854 & 1.6474 & 1.6435 & 1.6600 & 1.6525 \\
\hline$N V$ & 0 & 0 & 0 & 0 & 0 \\
\hline$N L$ & 0 & 0 & 0 & 0 & 0 \\
\hline
\end{tabular}

TABLE 9 Actual Load Model Scenario for Minimum MVA Configuration (DG of 1.43 p.u. at Bus 3) for Case 4: Error in Load Model

\begin{tabular}{|l|l|l|l|l|l|}
\hline & Const. & Res. & Ind. & Com. & Mix. \\
\hline$P_{L}$ & 0.0133 & 0.0139 & 0.0138 & 0.0141 & 0.0141 \\
\hline$Q_{L}$ & 0.0188 & 0.0125 & 0.0123 & 0.0125 & 0.0126 \\
\hline$P_{\text {intake }}$ & -0.0017 & 0.0230 & 0.0036 & 0.0387 & 0.0261 \\
\hline$Q_{\text {intake }}$ & 0.7118 & 0.7672 & 0.7956 & 0.7582 & 0.7680 \\
\hline$P_{\text {intake }}+P_{D G}$ & 1.4283 & 1.4530 & 1.4336 & 1.4687 & 1.4561 \\
\hline$S_{\text {intake }}$ & 0.7118 & 0.7675 & 0.7956 & 0.7592 & 0.7684 \\
\hline$M V A_{\text {sys }}$ & 1.6321 & 1.6431 & 1.6396 & 1.6529 & 1.6462 \\
\hline$N V$ & 0 & 0 & 0 & 0 & 0 \\
\hline$N L$ & 0 & 0 & 0 & 0 & 0 \\
\hline
\end{tabular}




\section{CONCLUSIONS}

A framework for testing the significance of load models on DG-location and sizing is developed and used to establish the importance of voltage-dependent behaviour of loads. Based on the study on 16-bus system following conclusions are drawn. Load models significantly affect the DG planning. It is established that DG planning based on constant power load models is not effective after implementation on actual systems. Decisions and results based on constant power load model assumptions are not technically feasible if employed on system having non-constant power load behavior.Significant effect of load models is observed on optimal location and size of DG resources in distribution system. A rise in reactive demand is observed when DG is placed in an actual system having non-constant power load model which is contrary to the observations in case of constant power load model assumption. It is shown that the though losses get reduced, the total power intake of the system increases which makes loss reduction redundant. It is established in this work that the quantities affected are (i) number of voltage limit violation, (ii) number of line limit violations (iii) MVA, real and reactive p.u demand on the main substation, (iv) loss reduction in real and reactive power (vi) saving of MVA capacity on the main substation (vii) optimal location and size of DG. It was observed that most or all of the quantities are affected.

\section{REFERENCES}

[1] Y. G. Hegazy, M. M. A. Salama, and A. Y. Chikhani, "Adequacy Assessment of Distributed Generation Systems Using Monte Carlo Simulation", IEEE Trans. Power Syst., vol. 18, no. 1, pp.48-52, Feb. 2003.

[2] Walid El-Khattam, Kankar Bhattacharya, Y. G. Hegazy, and M. M. A Salama, "Optimat Investment Planning for Distributed Generation in a Competitive Electricity Market”, IEEE Trans. Power Syst., vol. 19, no. 3, pp. 1674-1684, Aug 2004.

[3] Walid El-Khattam, Y. G. Hegazy, and M. M. A. Salama, "An Integrated Distributed Generation Optimization Model for Distribution System Planning", IEEE Trans. Power Syst., vol. 20, no. 2, pp. 1158-1165, May 2005.

[4] Caisheng Wang, and M. Hashem Nehrir, "Analytical Approaches for Optimal Placement of Distributed Generation Sources in Power Systems" IEEE Trans. Power Syst., vol. 19, no. 4, pp. 2068-2076 Nov 2004.

[5] Víctor H. Méndez Quezada, Juan Rivier Abbad and Tomás Gómez San Román, "Assessment of Energy Distribution Losses for Increasing Penetration of Distributed Generation", IEEE Trans. on Power Systems, Vol. 21, No. 2, pp. 533-540, May 2006.

[6] Paul M. Sotkiewicz and J. Mario Vignolo, "Allocation of Fixed Costs in Distribution Networks With Distributed Generation", IEEE Trans. on Power Systems, Vol. 21, No. 2, pp. 639-652, May 2006.

[7] C. Concordia and S. Ihara, "Load representation in power systems stability studies," IEEE Trans. Power App. Syst., vol. PAS-101, no. 4 pp.969-977, Apr. 1982.

[8] IEEE Task Force on Load Representation for Dynamic Performance, "Bibliography on load models for power flow and dynamic performance simulation," IEEE Trans. Power Syst., vol. 10, no. 1, pp. 523-538, Feb. 1995.

[9] IEEE Task Force on Load Representation for Dynamic Performance, "Load representation for dynamic performance analysis," IEEE Trans. Power Syst., vol. 8, no. 2, pp. 472-482, May 1993.

[10] IEEE Task Force on Load Representation for Dynamic Performance, "Standard load models for power flow and dynamic performance simulation," IEEE Trans. Power Syst., vol. 10, no. 3, pp. 1302-1313, Aug. 1995

[11] D.T. Rizy, J.S. Lawler, J.B. Patten, and W.R. Nelson, "Measuring and analyzing the impact of voltage and capacitor control with high speed data acqusition", IEEE Trans. on Power Delivery, Vol.4, No. 1, Jan 1989.

[12] Stefan Arnborg, Goran Anderson, David J. Hill, and Ian A. Hiskens, " On Influence of Load Modelling for Undervoltage Load Shedding
Studies", IEEE Trans. Power Syst., Vol. 13, No. 2, pp. 395-400, May 1998.

[13] Gozel, T., Hocaoglu, M.H. Eminoglu, and U. Balikci, A, "Optimal placement and sizing of distributed generation on radial feeder with different static load models" Proc. Int. Conf. Future Power Systems, pp. 1-6, Nov, 2005.

[14] M.E. Baran and F. F. Wu, "Network reconfiguration in distribution systems for loss reduction", IEEE Trans. on Power Delivery, vol. 4, no. 2, pp. 1401-1407, Apr. 1989.

[15] Dan Zhu, Robert P. Broadwater, Kwa-Sur Tam, Rich Seguin and Haukur Asgeirsson, "Impact of DG Placement on Reliability and Efficiency with Time-Varying Loads" IEEE Trans. On Power Systems, vol. 21, No. 1, pp. 419-427, Feb, 2006.]

[16] Rodrigo Palma-Behnke, José Luis Cerda A., Luis S. Vargas, and Alejandro Jofré, "A Distribution Company Energy Acquisition Market Model With Integration of Distributed Generation and Load Curtailment Options", IEEE Trans. on Power Systems, Vol. 20, No. 4, pp. 1718-1727, Nov 2005

[17] P S Nagendra Rao and Ravishankar Deekshit, "Energy Loss Estimation in distribution feeders", IEEE Trans. power delivery, vol. 21, No. 3, Jul 2006.

[18] Jorge E. Mendoza, Dario A. Morales, Rodrigo A. Lopez, Enrique A. Lopez, Lean-Claude Vannier and Carlos A. Coello Coello, "Multiobjective Location of Automatic Voltage Regulators in Radial Distribution Network Using a Micro Genetic Algorithm", IEEE Trans. on Power Systems, Vol. 22, No. 1, pp. 404-412, Feb 2007.

\section{APPENDIX 1}

TABLE A1 SYSTEM AND LOAD DATA FOR 16-BUS SYSTEM Line Impedance in p.u. Loads on

To-node (p.u)

\begin{tabular}{lllllllll}
\hline $\mathrm{F}$ & $\mathrm{T}$ & R p.u. & X p.u. & $\mathrm{L}$ & $\mathrm{S}_{\mathrm{L}}$ & $\mathrm{P}$ & $\mathrm{Q}$ & $\mathrm{L}_{\mathrm{T}}$ \\
\hline 1 & 2 & 0.000574 & 0.000293 & 1 & 1.92 & 0.1 & 0.06 & $\mathrm{R}$ \\
\hline 2 & 3 & 0.001021 & 0.000974 & 2 & 1.8 & 0.09 & 0.04 & $\mathrm{I}$ \\
\hline 3 & 4 & 0.009366 & 0.00844 & 3 & 1.2 & 0.12 & 0.08 & $\mathrm{C}$ \\
\hline 4 & 5 & 0.00255 & 0.002979 & 5 & 0.84 & 0.06 & 0.03 & $\mathrm{R}$ \\
\hline 4 & 6 & 0.004414 & 0.005836 & 6 & 0.78 & 0.06 & 0.02 & $\mathrm{I}$ \\
\hline 6 & 7 & 0.00307 & 0.001564 & 11 & 0.6 & 0.2 & 0.1 & $\mathrm{C}$ \\
\hline 7 & 8 & 0.002809 & 0.00192 & 7 & 0.54 & 0.2 & 0.1 & $\mathrm{C}$ \\
\hline 8 & 9 & 0.005592 & 0.004415 & 12 & 0.48 & 0.06 & 0.02 & $\mathrm{I}$ \\
\hline 8 & 10 & 0.005579 & 0.004366 & 13 & 0.42 & 0.06 & 0.02 & $\mathrm{C}$ \\
\hline 10 & 11 & 0.003113 & 0.003113 & 15 & 0.18 & 0.045 & 0.03 & $\mathrm{C}$ \\
\hline 3 & 12 & 0.002279 & 0.001161 & 9 & 0.18 & 0.06 & 0.035 & $\mathrm{R}$ \\
\hline 12 & 13 & 0.002373 & 0.001209 & 4 & 0.12 & 0.06 & 0.035 & $\mathrm{C}$ \\
\hline 13 & 14 & 0.0051 & 0.004402 & 8 & 0.12 & 0.12 & 0.08 & $\mathrm{R}$ \\
\hline 14 & 15 & 0.001264 & 0.000644 & 14 & 0.12 & 0.06 & 0.01 & $\mathrm{C}$ \\
\hline 14 & 16 & 0.00177 & 0.000901 & 16 & 0.12 & 0.06 & 0.02 & $\mathrm{I}$ \\
\hline 16 & 17 & 0.006594 & 0.005814 & 10 & 0.072 & 0.06 & 0.02 & $\mathrm{C}$
\end{tabular}

F=From node, $\mathrm{T}=$ To node, $\mathrm{L}=$ Line number, $\mathrm{S}_{\mathrm{L}}=$ Line MVA limit in p.u., $\mathrm{P}=$ Real MW load in p.u., $\mathrm{Q}=$ Reactive MVAr load in p.u., $\mathrm{L}_{\mathrm{T}}=\mathrm{Load}$ Type, $\mathrm{R}=$ Residential, $\mathrm{I}=$ Industrial, $\mathrm{C}=$ Commercial

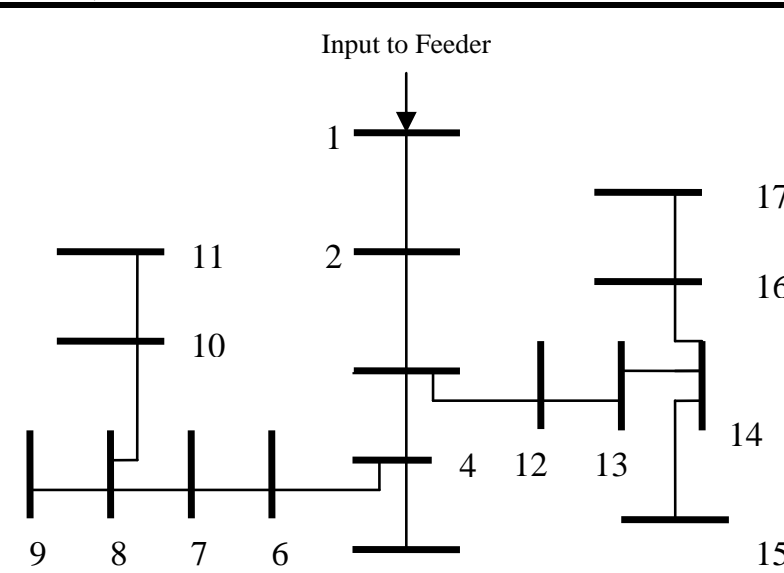


Fig. A1. 16-bus test system

Deependra Singh received the B. Tech and M.E degrees both in electrical engineering in 1997 and 1999 from Harcourt Butler Technological Institute, Kanpur, India and University of Roorkee, Roorkee, India, respectively. $\mathrm{He}$ is Assistant Professor in department of electrical engineering, Kamla Nehru Institute of Technology, Sultanpur (UP), India. Currently he is pursuing his Ph.D. from UP Technical University, Lucknow, India. His research interests are distributed generation planning and distribution system analysis.

Devender Singh received the B.E and M.E degrees both in electrical engineering in 1993 and 1999 from Sardar Vallabhbhai Regional College of Engineering and Technology, Surat, India and Motilal Nehru Regional Engineering College, Allahabad, India, respectively. He obtained his Ph.D in Electrical Engineering from Institute of Technology (IT), Banaras Hindu University (BHU), Varanasi, India. Presently, he is Reader in department of electrical engineering, IT, BHU, Varanasi, India. His research interests are distribution generation planning, state estimation, short term load forecasting, and AI applications in power systems.

K S Verma received the B. Tech and M.Tech. degrees both in electrical engineering from department of electrical engineering, Kamla Nehru Institute of Technology, Sultanpur (UP), India respectively. He obtained his Ph.D in Electrical Engineering from Indian Institute of Technology (IIT), Roorkee, Uttaranchal, India. Presently, He is Professor in department of electrical engineering, Kamla Nehru Institute of Technology, Sultanpur (UP), India. His research interests are FACTS, open power market, simulation and design of power systems, distributed generation planning. 